This Work has been submitted to Academic Press, Journal of Computational Physics For POSSIBLE PUBliCATION. COPYRIGHT MAY BE TRANSFERRED Without NOTICE, AFTER WHICH THIS VERSION MAY NO LONGER BE ACCESSIBLE.

\title{
Comparison of kinetic theory and discrete element schemes for modelling granular Couette flows
}

\author{
Lars Popken \\ Fachbereich Mathematik, Universität Kaiserslautern, 67653 Kaiserslautern, Germany. \\ e-mail: popken@mathematik.uni-kl.de \\ Paul W. Cleary \\ CSIRO Mathematical and Information Sciences, \\ Private Bag 10, Clayton South MDC, Clayton, VIC, 3169, Australia, \\ e-mail: Paul.Cleary@cmis.csiro.au
}

\begin{abstract}
Discrete element based simulations of granular flow in a $2 \mathrm{~d}$ velocity space are compared with a particle code that solves kinetic granular flow equations in two and three dimensions. The binary collisions of the latter are governed by the same forces as for the discrete elements. Both methods are applied to a granular shear flow of equally sized discs and spheres. The two dimensional implementation of the kinetic approach shows excellent agreement with the results of the discrete element simulations. When changing to a three dimensional velocity space, the qualitative features of the flow are maintained. However, some flow properties change quantitatively.
\end{abstract}

MSC: $65 \mathrm{C} 05,76 \mathrm{~F} 10,82 \mathrm{~B} 40,82 \mathrm{~B} 80$.

Keywords: Kinetic theory, discrete element method, granular flow, shear flow. 


\section{Introduction}

Flows of granular materials are widespread in our environment, for example in natural phenomena like avalanches or sand storms, or in industrial and technological processes, where bulk materials like grains, coal, ore, etc. are transported, screened or crushed.

The two major regimes in which motion of granular media occurs are rapid and slow flows. The latter ones are characterized by long duration contacts between particles during their motion. In this regime, bulk properties of moving granular media are controlled by the Coulomb inter-particle friction forces. In rapid flows on the other hand, particles move freely between successive collisions. Transfer of particle kinetic energy and momentum within a rapidly flowing granular medium occurs during these collisions and they govern the transport properties.

Particle impacts within granular materials are related to kinetic energy losses, associated with the inelasticity of collisions and the surface roughness. Hence, a constant source of mechanical energy is needed to sustain the motion of granular material.

Different approaches to modelling the dynamics of granular flow lead to different levels of description:

1. The microscopic level: Simulations of granular flow as an ensemble of a large number of rigid bodies are generally deterministic models. Every 'real' particle is represented by exactly one 'virtual' particle in a computer code. Particles move in a potential field and their interactions are defined by different models. The LennardJones potential leads to molecular dynamics simulations [1] while linear or Hertzian forces lead to soft particle discrete element methods [5,6]. Details of this method are described later in this paper. Recent applications of such methods include grinding ballmills [7] and the filling of dragline buckets [8].

2. The mezzoscopic level: Starting from an $N$-particle system described by the Liouville equation, a system of equations for $s$-particle systems $(s<N)$ may be derived. This so-called BBGKY hierarchy leads - in the limit $N \rightarrow \infty$ and under certain assumptions - to a kinetic equation for the one-particle density function in the phase space. A rigorous treatment for hard spheres yields the well-known Boltzmann equation. In the following, a derivative [11, 15] of the Boltzmann equation is considered, which takes into account the dense character of granular flows as well as the energy loss at impact.

3. The macroscopic level: The highest level of description consists of macroscopic field equations, generally partial differential equations. This is the more conventional scale for simulation but will not be considered in this work.

One of the essential characteristics of the kinetic ansatz is the binary type of collisions, i.e. exactly two particles are involved with any particle collision. After contact, all particles have to separate from each other again. The building of clusters and bridges is, at least in 
this framework, not possible. This means, that the problems considered have to be set in the rapid flow regime. Furthermore, gravity is neglected. A simple test case may already show differences between both methods. Hence, for the comparison of both methods we choose a problem that is $1 \mathrm{~d}$ in space, a Couette flow at a moderate packing density. Granular material is enclosed between two parallel walls. External energy is introduced by shearing the walls in opposite directions (Couette flow), where the distance between the walls remains constant. This shears the granular material setting up non-linear velocity and density distributions.

The present work is organized as follows. In the first part, an introduction to the kinetic formulation is given. A particle scheme to determine a solution of the kinetic equation is outlined. An introduction to the soft particle discrete element method (DEM) is given in the second part. A first simulation of a spatially homogeneous case gives the relationship between the mean free path and the solid fraction for different 'soft' collision parameters. In the third part both methods are applied to the Couette flow and the results compared, when the velocity space is two-dimensional. Comparisons of the $3 \mathrm{~d}$ kinetic particle scheme and the 2d DEM are given in the fourth section. In the last part, both methods are compared with respect to their calculations times.

\section{Formulation of the Kinetic Approach to Granular Flow}

A derivative of the Enskog [9] equation for dense gases is the following kinetic equation for granular flows [11]. It refers to a density $f$ in phase space, such that $f=f(t, \boldsymbol{x}, \boldsymbol{v}, \boldsymbol{\omega})$ is a scalar function of time $t$, position $\boldsymbol{x} \in \Lambda \subset \mathbf{R}^{3}$, velocity $\boldsymbol{v} \in \mathbf{R}^{3}$ and spin $\boldsymbol{\omega} \in \mathbf{R}^{3}$. We denote $\mathbf{w}=(\boldsymbol{v}, \boldsymbol{\omega})$ as the vector including both velocity and spin. The particles are considered as spheres with diameter $a$. Also, we do not restrict the model to energy conserving particle encounters. The kinetic equation for granular flow is then:

$$
\begin{gathered}
\frac{\partial f}{\partial t}+\boldsymbol{v} \cdot \nabla_{\boldsymbol{x}} f=J(f, f), \\
J(f, f)=\iint_{\mathbf{R}^{6} \times S^{2}} k\left(\boldsymbol{v}_{21}^{\prime \prime} \cdot \boldsymbol{\eta}\right) n\left(t, \boldsymbol{x}-\frac{a}{2} \boldsymbol{\eta}\right) f\left(t, \boldsymbol{x}, \boldsymbol{w}^{\prime \prime}\right) f\left(t, \boldsymbol{x}-a \boldsymbol{\eta}, \boldsymbol{w}_{*}^{\prime \prime}\right) d \boldsymbol{\kappa}(\boldsymbol{\eta}) d \boldsymbol{w}_{*}^{\prime \prime} \\
-\iint_{\mathbf{R}^{6} \times S^{2}} k\left(\boldsymbol{v}_{21} \cdot \boldsymbol{\eta}\right)\left(n\left(t, \boldsymbol{x}-\frac{a}{2} \boldsymbol{\eta}\right) f(t, \boldsymbol{x}, \boldsymbol{w}) f\left(t, \boldsymbol{x}-a \boldsymbol{\eta}, \boldsymbol{w}_{*}\right) d \boldsymbol{\kappa}(\boldsymbol{\eta}) d \boldsymbol{w}_{*}\right.
\end{gathered}
$$

together with the velocity transformation

$$
\left(\boldsymbol{w}, \boldsymbol{w}_{*}\right)=T_{\boldsymbol{\eta}}\left(\boldsymbol{w}^{\prime \prime}, \boldsymbol{w}_{*}^{\prime \prime}\right), \quad \text { where } \boldsymbol{w}_{*}=\left(\boldsymbol{v}_{*}, \boldsymbol{\omega}_{*}\right), \boldsymbol{w}^{\prime \prime}=\left(\boldsymbol{v}^{\prime \prime}, \boldsymbol{\omega}^{\prime \prime}\right), \text { etc. }
$$

The vector $\boldsymbol{v}_{21}=\boldsymbol{v}_{*}-\boldsymbol{v}$ is the relative velocity of two colliding particles, $\boldsymbol{\eta}$ is a unit vector in the direction of the line of centers of two spheres of diameter $a$ at the time of their impact and $\boldsymbol{\kappa}(\boldsymbol{\eta})$ is the probability measure on $S^{2}$. The collision integral $J(f, f)$ preserves the same binary structure of the corresponding Boltzmann term, but the colliding spheres 
occupy different positions in space. The collision frequency is modified by the factor $h^{(2)}$ which approximates the pair correlation function [16].

In the framework of the so-called Standard Enskog Theory (SET), $h^{(2)}$ is simply set equal to the equilibrium pair correlation evaluated at the point of contact. From the Carnahan and Starling [4] approximation of the equation of state, the total equilibrium pair correlation is found to be

$$
h^{(2)}(n)=\frac{2-\nu}{2(1-\nu)^{3}} .
$$

Here, $\nu$ denotes the solid fraction of the medium, e.g. $\nu=\frac{n}{6} \pi a^{3}$ for hard spheres, where $n$ is the number density. In the limit $\nu \rightarrow 0$, the correlation $h^{(2)}(v)$ tends to one.

Up to now, it is not clear which equation of state is the most suitable one for granular flow. As we want to make clear the relation of (1) to the Enskog equation, in this work the pair correlation is always taken from SET. For a survey of different pair correlations in granular flow see, e.g. Goldshtein et al. [12].

The factor $k($.$) in (1) is the collision kernel for hard spheres, k\left(\boldsymbol{v}_{21} \cdot \boldsymbol{\eta}\right)=4 \pi a^{2}\left(\boldsymbol{v}_{21} \cdot \boldsymbol{\eta}\right) \theta\left(\boldsymbol{v}_{21}\right.$. $\boldsymbol{\eta})$ where $\theta$ is the Heaviside function.

The velocity transformation conserves linear and angular momenta, yet in order to find the post-collisional velocities $\left(\boldsymbol{w}, \boldsymbol{w}_{*}\right)=T_{\boldsymbol{\eta}}\left(\boldsymbol{w}^{\prime \prime}, \boldsymbol{w}_{*}^{\prime \prime}\right)$, one needs further relations. For hard spheres, where the binary collisions are instantaneous, one commonly relates the post-collisional relative velocity to the pre-collisional one [11]. If $\left(\boldsymbol{v}_{21} \cdot \boldsymbol{\eta}\right)>0$, then the relative velocity at the contact point before collision is given by:

$$
\boldsymbol{g}_{21}=\left(\boldsymbol{v}_{*}-\frac{a}{2} \boldsymbol{\eta} \times \boldsymbol{\omega}_{*}\right)-\left(\boldsymbol{v}+\frac{a}{2} \boldsymbol{\eta} \times \boldsymbol{\omega}\right)=v_{\eta} \boldsymbol{\eta}+\boldsymbol{v}_{\tau},
$$

where $v_{\eta}=\left(\boldsymbol{v}_{21} \cdot \boldsymbol{\eta}\right)$ is the modulus of the normal component of the relative velocity and $\boldsymbol{v}_{\tau}$ is its tangential component,

$$
\boldsymbol{v}_{\tau}=\boldsymbol{v}_{21}-\boldsymbol{\eta}\left(\boldsymbol{v}_{21} \cdot \boldsymbol{\eta}\right)-\frac{a}{2} \boldsymbol{\eta} \times\left(\boldsymbol{\omega}+\boldsymbol{\omega}_{*}\right) .
$$

After collision the velocity components are:

$$
\begin{gathered}
v_{\eta}^{\prime}=-e v_{\eta}, \\
\boldsymbol{v}_{\tau}^{\prime}=-\beta \boldsymbol{v}_{\tau},
\end{gathered}
$$

where the two coefficients $e$ and $\beta$ characterize the collision process. $e$ is the coefficient of restitution or inelasticity in the normal direction, $0<e \leq 1$, and $\beta$ is the roughness coefficient in the tangential direction, $-1 \leq \beta \leq 1$. For perfectly rough spheres one has $e=\beta=1$, whereas $e=1, \beta=-1$ for perfectly smooth spheres. These post-collisional velocities are then uniquely determined [11].

With the help of the one-particle distribution $f(t, x, w)$, the macroscopic moments of $f$ are obtained: The number density $n$, the bulk velocity $u$, the inner translational energy 
$e_{v}$ and the inner rotational energy $e_{\omega}$ :

$$
\begin{aligned}
n(t, \boldsymbol{x}) & =\int f(t, \boldsymbol{x}, \boldsymbol{w}) d \boldsymbol{w}, \\
\boldsymbol{u}(t, \boldsymbol{x}) & =\frac{1}{n} \int \boldsymbol{v} f(t, \boldsymbol{x}, \boldsymbol{w}) d \boldsymbol{w}, \\
e_{v}(t, \boldsymbol{x}) & =\frac{m}{2 n} \int|\boldsymbol{v}-\boldsymbol{u}|^{2} f(t, \boldsymbol{x}, \boldsymbol{w}) d \boldsymbol{w}, \\
e_{\omega}(t, \boldsymbol{x}) & =\frac{I}{2 n} \int|\boldsymbol{\omega}-\overline{\boldsymbol{\omega}}|^{2} f(t, \boldsymbol{x}, \boldsymbol{w}) d \boldsymbol{w} .
\end{aligned}
$$

In the above, $I$ denotes the moment of inertia of a particle and $\overline{\boldsymbol{\omega}}$ the mean spin.

\subsection{A Particle Method for the kinetic granular flow equation}

The solution method for the kinetic granular flow equation (1) is explained in detail in Popken [15]. It is a derivative of the finite pointset method (FPM) developed at the University of Kaiserslautern to solve the Boltzmann equation. The following FPM is based on the time splitting of (1).

Introducing two fractional time steps one solves first the free transport equation in $[0, \Delta t]$ :

$$
\frac{\partial f}{\partial t}+\boldsymbol{v} \cdot \nabla_{\mathbf{x}} f=0
$$

If the particle approximation of the initial value $f(0, \boldsymbol{x}, \boldsymbol{w})$ of $(11)$ is given by some discrete measure $\frac{1}{N} \sum_{j=1}^{N} \delta_{\mathbf{x}_{j}} \delta_{\mathbf{w}_{j}}$, then the time evolution of the particle ensemble is simply

$$
\frac{1}{N} \sum_{j=1}^{N} \delta_{\boldsymbol{x}_{j}+\Delta t} \boldsymbol{v}_{j} \delta_{\boldsymbol{w}_{j}}
$$

Boundary conditions are taken into account during the free flow, too. Collisions with the wall are modeled via the same laws and parameters as a regular particle-particle collision, see Eqs. (5)-(6).

In a second step the time dependent kinetic granular equation without free flow,

$$
\frac{\partial f}{\partial t}=J(f, f)
$$

is solved. To simulate Eq. (13) via a particle method, an explicit Euler step is used and Eq. (13) is written in its discretized form:

$$
f(\Delta t, \boldsymbol{x}, \boldsymbol{w})=f(0, \boldsymbol{x}, \boldsymbol{w})+J[f, f](0, \boldsymbol{x}, \boldsymbol{w}) .
$$

$f(\Delta t, \boldsymbol{x}, \boldsymbol{w})$ is then used in the next time step as the new initial condition for the free flow. Eq. (14) is now considered in a weak formulation, i.e. it is multiplied by a continuous test 
function $\varphi: \Lambda \times \mathbf{R}^{6} \rightarrow \mathbf{R}$ and integrated over the phase-space $\Lambda \times \mathbf{R}^{6}$. This leads to:

$$
\begin{aligned}
\iint_{\Lambda \times \mathbf{R}^{6}} \varphi(\mathbf{x}, \mathbf{w}) f(\Delta t, \mathbf{x}, \mathbf{w}) d \mathbf{w} d \mathbf{x} & = \\
\iiint \int_{\Lambda \times \mathbf{R}^{6} \times \mathbf{R}^{6} \times S^{2}}\left[\Delta t k(.) h^{(2)}(.) \varphi\right. & \left.\left(\boldsymbol{x}, \boldsymbol{w}^{\prime}\right)+\left(\frac{1}{n(0, \boldsymbol{x}-a \boldsymbol{\eta})}-\Delta t k(.) h^{(2)}(.)\right) \varphi(\boldsymbol{x}, \boldsymbol{w})\right] \\
& \times \quad f(0, \boldsymbol{x}, \boldsymbol{w}) f\left(0, \boldsymbol{x}-a \boldsymbol{\eta}, \boldsymbol{w}_{*}\right) d \boldsymbol{\kappa}(\boldsymbol{\eta}) d \boldsymbol{w}_{*} d \boldsymbol{w} d \boldsymbol{x},
\end{aligned}
$$

In such a particle scheme if one particle is located at a position $\boldsymbol{x}$, then in general no particle can be found at $\boldsymbol{x}-\boldsymbol{a} \boldsymbol{\eta}$, the position of the collision partner. Therefore, a collision partner is allowed to be at a position $\boldsymbol{x}_{*}$ close to $\boldsymbol{x}-a \boldsymbol{\eta}$. This idea is known as mollifying and details are contained in $[19,14]$. To solve the mollified version of Eq. (15) we need an approximation of the product measure:

$$
f(0, \boldsymbol{x}, \boldsymbol{w}) f\left(0, \boldsymbol{x}_{*}, \boldsymbol{w}_{*}\right) d \boldsymbol{\kappa}(\boldsymbol{\eta}) d \boldsymbol{w}_{*} d \boldsymbol{x}_{*} d \boldsymbol{w} d \boldsymbol{x},
$$

given only an approximation of $f(0, \boldsymbol{x}, \boldsymbol{w}) d \boldsymbol{w} d \boldsymbol{x}$ [13]. Then one can compute the time evolution of the measure due to (15): The factor $1-\Delta t k(.) h^{(2)}() n.(0, \boldsymbol{x}-\boldsymbol{a} \boldsymbol{\eta})$ may be interpreted as the probability of a dummy collision, keeping the old velocities and spins. $\Delta t k(.) h^{(2)}() n.(0, \boldsymbol{x}-a \boldsymbol{\eta})$ is the probability of a real collision, changing $\left(\boldsymbol{w}, \boldsymbol{w}_{*}\right) \rightarrow$ $\left(\boldsymbol{w}^{\prime}, \boldsymbol{w}_{*}^{\prime}\right)=T_{\boldsymbol{\eta}}\left(\boldsymbol{w}, \boldsymbol{w}_{*}\right)$. This is also the restriction on the time step. Eq. (15) is only sensible in the sense of measures, if the time step fulfills

$$
\Delta t k(.) h^{(2)}(.) \leq \frac{1}{n(t, .)}
$$

for all times $t$, all relative velocities $\boldsymbol{v}_{21}$ and all positions $x$. This means, that the time step is determined by the maximum inner translational energy $e_{v}$ and the maximum density $n$ that might occur in the flow.

The accuracy of the FPM has, in the rarefied regime, successfully been checked against some results of Bird's [3] well known DSMC-code, see e.g. [20]. The numerical convergence of the present test case is of order $O\left(N^{-1}\right)$, where $N$ is the number of particles. This is

described later on. For more details about the FPM we refer to the above cited references.

\section{Formulation of the Discrete Element Model for Granular Flow}

Modelling a force that represents an inelastic collision requires at least two terms: repulsion and some sort of dissipation. The simplest force with the desired properties is the damped harmonic oscillator shown in Figure 1.

The normal force $F_{\eta}$ has a spring component to provide the repulsive force that pushes the particles apart, and a dashpot that provides dissipation, resulting in an effective coefficient 


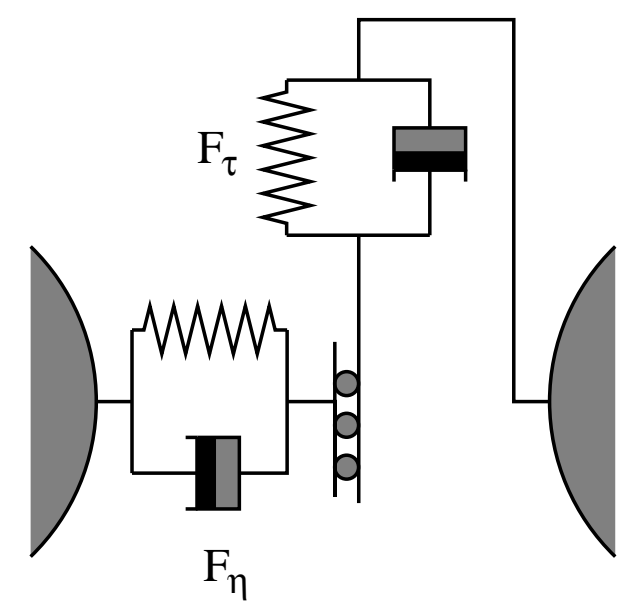

Figure 1: The spring-dashpot model. The normal force $F_{\eta}$ and the tangential force $F_{\tau}$ both use a spring and a dashpot. The tangential force is also limited by friction.

of restitution. The tangential component is again modeled by a spring and a dashpot, yet it is subject to the frictional limit of $\mu F_{\eta}$, where $\mu$ is the dynamic friction coefficient. Contact force models are discussed in review articles by [2, 21]. In the following, only mono-disperse spheres are considered, where the mass is denoted by $m$ and the moment of inertia by $I$.

The equations for the forces are:

$$
\begin{array}{ll}
\text { Normal Force : } & F_{\eta}=-c_{\eta} v_{\eta}-k_{\eta} \xi \\
\text { Tangential Force : } & F_{\tau}=-\min \left\{\mu\left|F_{\eta}\right|, k_{\tau} \int_{t_{0}}^{t} v_{\tau} d t^{\prime}+c_{\tau} v_{\tau}\right\},
\end{array}
$$

where $c_{\eta}, c_{\tau} \geq 0$ are the normal and the tangential damping coefficients (representing the dashpot), $k_{\eta}, k_{\tau} \geq 0$ are the normal and tangential spring constants and $\mu \geq 0$ is the friction coefficient. $v_{\eta}, v_{\tau}$ are the (scalar) velocities in the normal and tangential directions with $v_{\eta}(0)>0$ and $v_{\tau}(0) \geq 0$.

The spring elongation $\xi \geq 0$ is the actual overlap of the two particles. The spring in the tangential direction is loaded by the relative tangential movement of the particles' surfaces, given by integration of $v_{\tau}$ over the time from the onset $t_{0}$ of the collision until time $t$. The initial conditions are $\xi(0)=0$ and $v_{\eta}(0)=\boldsymbol{v}_{21} \cdot \boldsymbol{\eta}>0$, where $\boldsymbol{v}_{21}=\boldsymbol{v}_{2}-\boldsymbol{v}_{1}$ is the relative velocity between two colliding particles at position $\boldsymbol{x}_{2}$ and $\boldsymbol{x}_{1}$ at the beginning of a collision. The unit vector $\boldsymbol{\eta}$ points from $\boldsymbol{x}_{2}$ to $\boldsymbol{x}_{1}$.

Newton's Law:

$$
m_{*} \ddot{\xi}=F_{\eta}
$$


with $m_{*}=m / 2$ being the reduced mass, can easily be solved when $k_{\eta}>c_{\eta}^{2} / 4 m_{*}$ to give:

$$
\xi(t)=\frac{v_{\eta}(0)}{\gamma} \exp \left(-\frac{c_{\eta}}{2 m_{*}} t\right) \sin (\gamma t)
$$

with

$$
\gamma=\sqrt{\frac{k_{\eta}}{m_{*}}-\frac{c_{\eta}^{2}}{4 m_{*}^{2}}} .
$$

The encounter time $t_{*}$ that elapses during a collision is $t_{*}=\pi / \gamma$. If an inelasticity $e \in[0,1]$ as defined in (5) is given, then $c_{\eta}$ is found to be:

$$
c_{\eta}=-2 \sqrt{m_{*} k_{\eta}} \cdot \frac{\ln (e)}{\sqrt{\pi^{2}+\ln (e)^{2}}},
$$

with $\ln ($.$) the natural logarithm.$

The scalar forces $F_{\eta}$ and $F_{\tau}$ still have to be transformed into the global coordinate system. The resulting force of a particle $j$ acting on particle $i$ in the global coordinate system is denoted by $\boldsymbol{F}_{j i}$.

The equations of motion for the particles are then the following ( $i=1,2$ for a binary collision).

$$
\begin{aligned}
\dot{\boldsymbol{x}}_{i} & =\boldsymbol{v}_{i} \\
\dot{\boldsymbol{v}}_{i} & =\frac{1}{m} \sum_{j} \boldsymbol{F}_{j i} \\
\dot{\boldsymbol{\omega}}_{i} & =\frac{1}{I} \sum_{j} \boldsymbol{M}_{j i} .
\end{aligned}
$$

To find the resulting forces $\boldsymbol{F}_{j i}$ we assume that the unit vector $\boldsymbol{\eta}$ points from $\boldsymbol{x}_{j}$ to $\boldsymbol{x}_{i}$. The unit vector $\boldsymbol{\tau}$ is orthogonal to $\boldsymbol{\eta}$ throughout a collision, initially pointing into the direction of the tangential velocity vector $\boldsymbol{v}_{\tau}$, see Eq. (4).

The force $\boldsymbol{F}_{j i}$ is composed of $F_{\eta}$ and $F_{\tau}$, the relative forces in the binary collision, via

$$
\boldsymbol{F}_{j i}= \begin{cases}-F_{\eta} \boldsymbol{\eta}+F_{\tau} \boldsymbol{\tau}, & \text { for }\left|\boldsymbol{x}_{i}-\boldsymbol{x}_{j}\right| \leq a \text { and } i \neq j \\ 0, & \text { else. }\end{cases}
$$

It follows, that $\boldsymbol{F}_{j i}=-\boldsymbol{F}_{i j}$ holds. The torque is defined as

$$
\boldsymbol{M}_{j i}=-\frac{a}{2} \boldsymbol{F}_{\tau}(\boldsymbol{\eta} \times \boldsymbol{\tau}),
$$

and $\boldsymbol{M}_{j i}=\boldsymbol{M}_{i j}$.

The choice of the tangential parameters $k_{\tau}$ and $c_{\tau}$ determine the frequency of the tangential oscillation. Yet, the tangential force in (19) is coupled to the normal force (both forces 
accelerate the centres of mass, hence the change of $v_{\tau}$ also depends on $F_{\eta}$ ). A general analytic solution to the system (24)-(26) is not known to us.

Since the encounter time $t_{*}>0$ is now finite, collisions of three or more particles in a flow field are possible. The bigger the encounter time, the larger the amount of triple or quadruple collisions.

In the present code, the equations of motion (24)-(26) are solved by an explicit time stepping scheme, where the time step $\Delta t$ is set to:

$$
\Delta t=\frac{t_{*}}{25}
$$

Before applying the above methods to the Couette flow test case, the DEM is applied to the homogeneous case. By tracing the mean free path, the pair correlation function is determined for different spring parameters.

\section{Application of the 2D-DEM to the Homogeneous Case}

A characteristic feature of dense hard sphere gases is that, for a solid fraction $\nu \gg 0$, the mean free path $\lambda$ is no longer proportional to the inverse of the number density of the flow. An additional factor comes into play, that originates in the pair correlation evaluated at the contact point of a binary collision.

For a gas at equilibrium, the mean free paths are known to be

$$
\begin{aligned}
\lambda_{2 d} & =\frac{\sqrt{2}}{4 n a h_{2 d}^{(2)}(n)}, \\
\lambda_{3 d} & =\frac{1}{\sqrt{2} n \pi a^{2} h_{3 d}^{(2)}(n)} .
\end{aligned}
$$

To determine the equilibrium mean free path, knowledge about the pair correlation function $h^{(2)}$ is required, which is gained from the equation of state. The equivalent of $(2)$ in the case of hard discs [17] leads to

$$
h_{2 d}^{(2)}(\nu)=\frac{2-\nu}{2(1-\nu)^{2}},
$$

where $\nu=\frac{1}{4} \pi a^{2} n$ for discs.

Eq. (30) is now used to check the DEM for a homogeneous flow of smooth elastic discs. Two problem sizes are used with 400 and 1000 discs respectively. They are placed in a square box with periodic boundaries in each direction. The size of the box determines the solid fraction. In these simulations the parameters of the soft particle collision model are set to $k_{\eta}=10,000 \mathrm{~N} / \mathrm{m}$, the mass $m=0.96 \mathrm{~kg}$ and $c_{\eta}=\mu=0$. The initial velocity of the 


\begin{tabular}{|r|c|c|}
\hline$k_{\eta} / m$ in $s^{-2}$ & mean overlap & maximal overlap \\
\hline \hline 10,416 & $0.9 \%$ & $5.0 \%$ \\
\hline 1,042 & $2.6 \%$ & $15.2 \%$ \\
\hline 104 & $7.9 \%$ & $56.7 \%$ \\
\hline
\end{tabular}

Table 1: The mean and the maximal overlap for different parameter ratios and a constant mean relative velocity $\overline{\boldsymbol{v}}_{21} \approx 0.59 a / \mathrm{s}$.

particles is such that the mean relative normal velocity vector $\overline{\boldsymbol{v}}_{\eta}$, defined by:

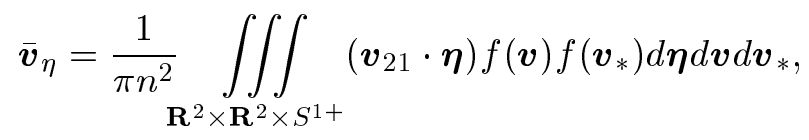

is given as $\overline{\boldsymbol{v}}_{\eta} \approx 0.59 \mathrm{a} / \mathrm{s}$, in the units of disc diameters per second. In the above, $S^{1+}$ is the half unit circle with $\left(\boldsymbol{v}_{21} \cdot \boldsymbol{\eta}\right) \geq 0$.

The resulting mean overlap of the binary collisions is around $0.9 \%$, i.e. the collisions are not very soft, but rather close to a hard disc collision. The maximal overlap encountered in the simulations is less than $5 \%$. The mean free path between collisions is calculated and gives the pair correlation from (30). In Figure 2, the 2d pair correlation from the DEM simulation (denoted by ' $\mathrm{o}$ ') is compared to the $2 \mathrm{~d}$ hard disc pair correlation (the solid line) calculated using Eq. (32). This is the instantaneous hard collision limit as $k_{\eta} \rightarrow \infty$.

The agreement of this data to that of the hard disc correlation is very good with only a small deviation appearing for high solid fractions $\nu_{2 d} \geq 0.55$. The deviation increases with solid fraction because an increasing proportion of particles are involved in collisions at any time. A mean overlap of less than $1 \%$ is sufficient to ensure that results are close to those of hard disc collisions.

Next, the mean free path is measured for softer collisions, when the mean particle overlap is $2.6 \%$ and $7.9 \%$ for $k_{\eta} / m=1,042 s^{-2}$ and $k_{\eta} / m=104 s^{-2}$, respectively. The normal relative velocity $\overline{\boldsymbol{v}}_{21}$ is the same as in the previous case. Table 1 shows the mean and maximum soft particle overlap obtained from the DEM for various parameter ratios $k_{\eta} / m$.

The corresponding correlation functions for the larger overlaps are shown in Figure 2 and denoted by ' + ' and ' $*$ ' symbols. For an overlap of $2.6 \%$, the difference between the soft and hard disc correlation is small for $\nu_{2 d} \leq 0.2$, then increases with the solid fraction. For an overlap of $7.9 \%$ the correlation differs substantially from the hard particle one for all but the lowest solid fractions.

Theoretically, the derivation of the mean free path in Eq. (30) is only valid for hard disc potentials. But, as a first approximation, it gives suitable correlation functions for soft encounters. Decreasing the spring constant $k_{\eta}$ increases the collision time $t_{*}$ and therefore the mean overlap. This produces more space for the other particles leading to an increase in the mean free path and a corresponding decrease in the correlation function.

The mean overlap, which depends on the mean normal relative velocity $\overline{\boldsymbol{v}}_{21}$ and the spring 


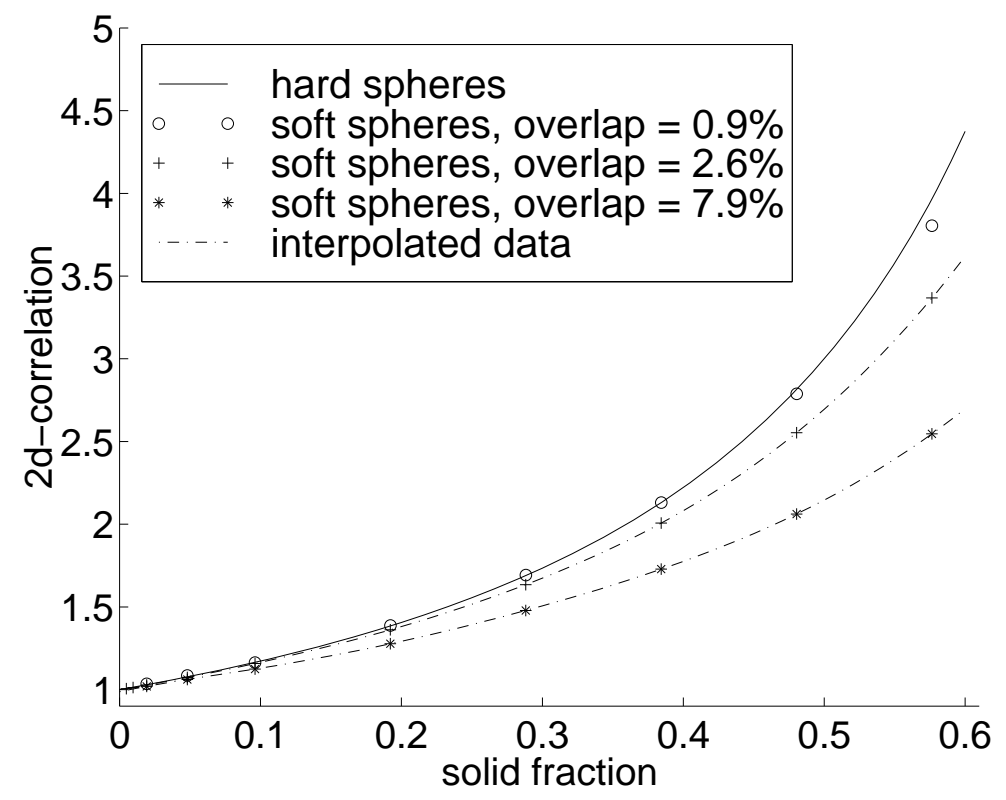

Figure 2: The 2d correlation function $h_{2 d}^{(2)}$ at contact for different mean overlaps.

to mass ratio $k_{\eta} / m$, is the essential parameter that determines how close the characteristics of a soft sphere flow are to a hard sphere one. This confirms that the typical $1 \%$ overlap criterion used in DEM applications is adequate to ensure that the particles behave as hard particles.

It is important to recall that for an increasing encounter time $t_{*}$, triple and multi-body collisions are more and more frequent. Hence, if the above correlation functions for soft encounters are to be used in a kinetic formulation such as the kinetic granular flow equation for hard spheres, Eq. (1), one has to check the validity of the binary collision ansatz. In the following chapter we assume that the encounter time $t_{*}$ is small in comparison to the mean time between collisions. We take this as an argument that the binary model is still sufficiently well-suited.

\section{The Comparison Case: A Granular Couette Flow}

A dense granular particle ensemble is situated between two parallel oppositely moving plates. Without an energy source, the ensemble would converge toward a zero granular temperature state. In a Couette flow, energy is constantly supplied to the system by the shearing. Figure 3 shows the Couette flow configuration used in these simulations. Particle-wall and particle-particle collisions are modelled according to the spring-dashpot model (18)-(19). In subsequent simulations, both hard sphere and hard disc, the moment of inertia $I$ is set to be the one for discs, $I=m a^{2} / 8$, thus providing a constant base for 
comparison.

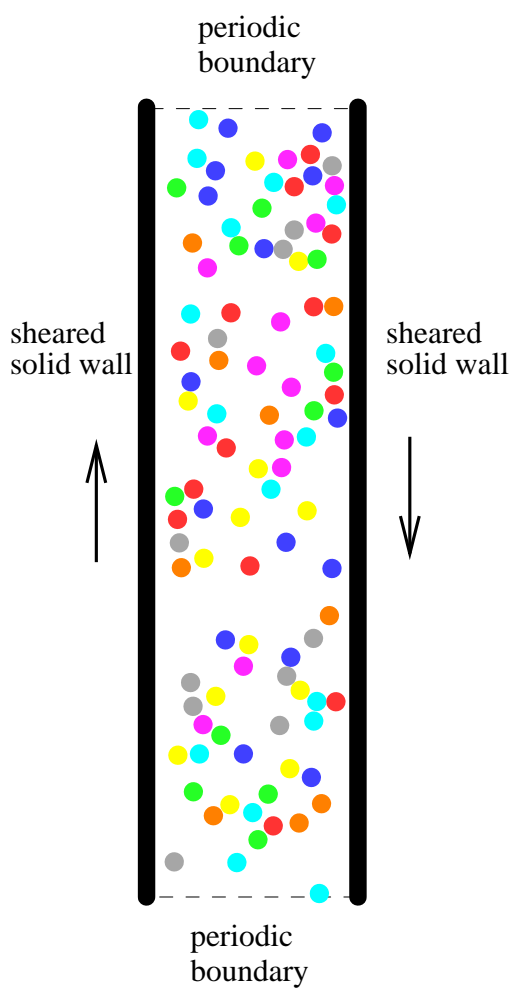

Figure 3: The Couette flow configuration.

\subsection{Convergence to Steady State and Data Averaging}

The particles are started with random positions and velocities with an average of $0.59 a$. The first flow regime is transient with the particles re-arranging, exchanging momentum and energy, and evolving towards a steady state. A criterion is needed to determine when the transient regime has ended and the profiles have become stationary. We have found that among the first and second moments, such as density, bulk velocity, bulk spin and inner energy, some of the profiles become stationary later than the others. In particular, the transients of the inner energy vanish last for the Couette flow. Therefore, we calculate the spatial mean of the inner energy $e_{v}+e_{\omega}$ and smooth it over a short length of time. The time interval of averaging increases with time, thus reducing the influence of the noise. In Figure 4, the convergence for the FPM to steady state is shown. The time is scaled by the mean time between collisions $t_{m}$, which is the ratio of the mean free path $\lambda$ and the initial most probable speed $c_{0}$. The mean of the sum of $e_{v}$ and $e_{\omega}$ is scaled to $\frac{3}{4} m c_{0}^{2}$.

Here, the first averaging is done over 5 time units, then over 10, 15, 20, etc. This averaging is necessary to eliminate the noise in the inner energy that arises from the energy transfer 


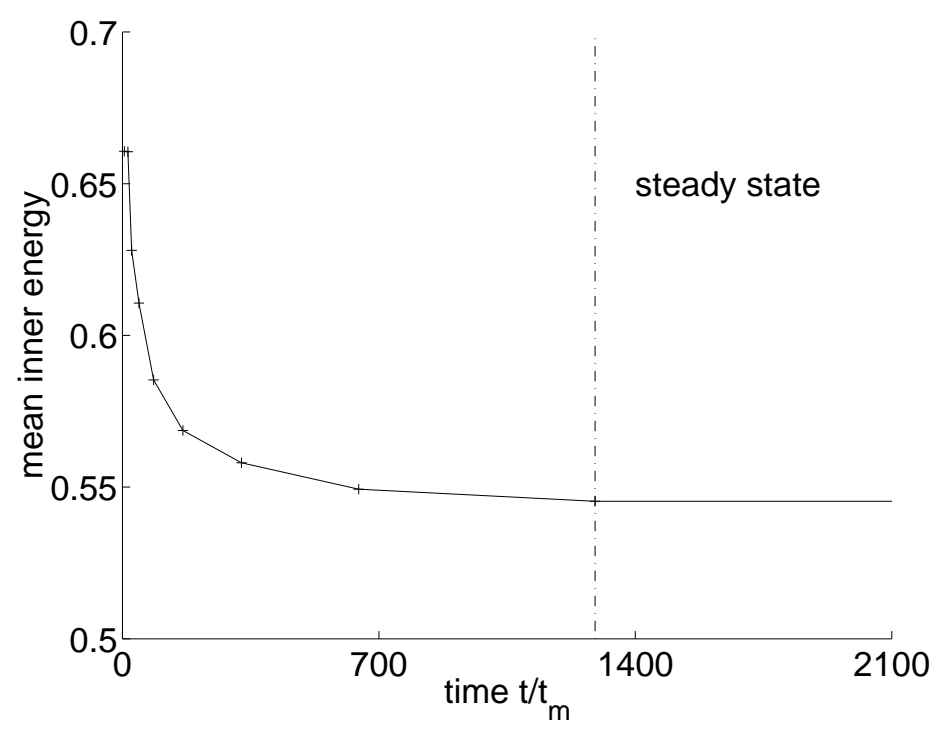

Figure 4: Convergence of the mean inner energy with time.

between the particles and the energy of the springs in the collision model. After 1,290 time units, the inner energy has sufficiently converged and the flow is considered to be stationary.

Data is not collected on every time step since the particles move only small distances between time steps and thus this data is highly correlated. After $t_{m}$, the average time between collisions, the particles have all moved to new positions and experience different collisions. The data used for averaging is therefore sampled every $t_{m}$ time units to ensure that this data is statistically uncorrelated.

The length of the averaging interval determines the number of sets of data used in the averaging. The average of the data at a specific time varies from the underlying asymptotic flow by an amount which decreases with the amount of data. If $t_{s}$ denotes the time when the flow field has become stationary, then the first averaging is over the interval $\left[t_{s}, t_{s}+10\right]$, then over $\left[t_{s}, t_{s}+20\right],\left[t_{s}, t_{s}+40\right]$ and so on. Let $N_{t}$ denote the number of times at which uncorrelated data is sampled. The standard deviation, which is a measure for the noise of the profiles, is then of the order of $1 / \sqrt{N_{t}}$. In particular, in the following simulations the averaging takes place over 5,120 points in time, leading to maximum errors in the profiles of the order of $1.4 \%$.

\subsection{Numerical Order of Convergence for the FPM}

The profiles of the stationary solution may vary slightly with the number of particles $N$ per cell used in the simulation. We found that the number density as well as the bulk velocity are independent of $N$. However, second moments like $e_{v}$ and $e_{\omega}$ are, for initially 
$N=10$ per cell (using 10 cells to resolve the distance of one mean free path), around $10 \%$ lower than the respective profiles for $N \rightarrow \infty$.

To find the order of convergence of the latter profiles, we compare the stationary, timeaveraged energy profiles of the inner translational energy $e_{v}^{N}$, depending on the number of particles $N$ per cell. If we assume that the $L^{\infty}(\Lambda)$ norm of the difference of $e_{v}^{N}$ and the asymptotic solution $e_{v}^{\infty}$ converges with order $k$ for $N \rightarrow \infty$,

$$
\left\|e_{v}^{N}-e_{v}^{\infty}\right\|_{L^{\infty}}=O\left(N^{-k}\right)
$$

then we define the numerical rate of convergence $k$,

$$
k:=\log _{j} \frac{\left\|e_{v}^{N}-e_{v}^{j N}\right\|_{L^{\infty}}}{\left\|e_{v}^{j N}-e_{v}^{j^{2} N}\right\|_{L^{\infty}}},
$$

where $j$ is a natural number. From the simulations for the Couette flow a numerical rate of convergence of $k \approx 1$ is found, i.e. for $N=100$ particles, the energy profile $e_{v}^{N}$ is within a $1 \%$ range of the asymptotic solution $e_{v}^{\infty}$.

\section{Comparison of Couette Flow Results in 2D}

\subsection{Considerations on the Time Step}

A critical point for an objective comparison of the two methods is the choice of the time step. The time step in the FPM is, for a bounded collision kernel $k($.$) , only dependent on$ the inner energy $e_{v}(t)$. If, for a proper comparison, the mean overlap in the DEM is to be kept less than $1 \%$, then the parameter ratio $k_{\eta} / m$ has to be appropriate for the inner energy $e_{v}$. The higher $e_{v}$ is the higher has to be $k_{\eta} / m$. Accordingly, the encounter time $t_{*}$ decreases.

The time step for the DEM has to accurately integrate the binary soft particle collision (see Eq. (29)). For a parameter ratio of $k_{\eta} / m=10,416 \mathrm{~s}^{-2}$, the time step $\Delta t_{s}$ of the spring-dashpot model has to be approximately 40 times smaller than the time step $\Delta t_{k}$ of the FPM. This immense difference in the time steps is due to the main characteristic of both schemes. Where the DEM has to integrate the equations of motion for all collisions in a deterministic way, the FPM lets the particles move freely for some time (about 1/20 of the mean time between collisions) and determines the effects of collisions afterwards using probabilistic means.

\subsection{The Choice of Parameters for Comparison}

For the DEM a set of 100 discs are used, while 10,000 particles are for the FPM. The initial solid fraction is chosen as $\nu_{2 d}=0.213$ and the distance between the plates is set to $L=10 \lambda+a$, so that the width of the flow field is 10 mean free paths. 
Every collision in this DEM simulation dissipates energy according to the spring-dashpot model with spring-mass ratio $k_{\eta} / m=10,416$. The ratio of the tangential to the normal spring constant is set to $k_{\tau} / k_{\eta}=1 / 3$ and the friction coefficient $\mu=10^{6}$, thus eliminating sliding of the surfaces. The above choice of the spring constants in normal and tangential direction means that for an elastic $\left(c_{\eta}=c_{\tau}=0\right)$ and rough $(\mu=\infty)$ binary collision, the collision frequency in the normal direction is equal to the collision frequency in tangential direction (neglecting any effect of the disc overlap). This means, that when the particles separate at the end of the collision, the tangential spring is unloaded at the same time and no energy is lost.

For $e<1$ and a finite overlap, the tangential spring stiffness is still chosen in the same way. Hence, there is a very small additional energy loss, as the energy that is still stored in this spring at the time of separation in the normal direction is not converted back into particle motion. Here, we choose the inelasticity to be $e=0.95$.

The inelasticity $e$ defines the damping coefficient $c_{\eta}$ of the normal spring via (23). As we have no rule how to set the damping coefficient $c_{\tau}$ in tangential direction, we take

$$
c_{\tau}=c_{\eta} \sqrt{\frac{k_{\tau}}{k_{\eta}}},
$$

which relates $c_{\tau}$ to $k_{\tau}$ and $e$ in the same manner as for $c_{\eta}$ in (23). However, the corresponding restitution coefficient $\beta$ is smaller than $e$, since the tangential spring is still loaded when the particles separate. For $e=0.95$, the above ratio of the spring constant (numerically) leads to $\beta \approx 0.915$. As the friction coefficient is chosen very high, $\beta$ is constant for all pre-collisional velocities.

The constant shear velocities of the walls are set to be the initial most probable particle speed $c_{0}$. All velocities are normalized by $c_{0}$. The position $x$ in the spanwise direction across the shear flow is normalized to the initial mean free path $\lambda_{0}$. Other variables are normalized accordingly.

The collision kernel $k($.$) of the collision operator as well as the pair correlation are here$ taken as the ones from hard disc theory, i.e. $k_{2 d}\left(\boldsymbol{v}_{21} \cdot \boldsymbol{\eta}\right)=2 \pi a\left(\boldsymbol{v}_{21} \cdot \boldsymbol{\eta}\right) \theta\left(\boldsymbol{v}_{21} \cdot \boldsymbol{\eta}\right)$ and $h_{2 d}^{(2)}$ as in (32).

\subsection{Comparison of DEM and FPM flows in $2 \mathrm{~d}$}

After the initial transient regime, stationary profiles develop. The mean overlap is around $0.8 \%$ and hence the soft particles behave like hard ones. The bulk velocity $u_{y}$ parallel to the walls is shown in Figure 5 for both profiles. The profiles agree very closely. Both curves show the same subtle change in the flow at the distance of one particle diameter from the walls indicating the existence of a boundary layer. The boundary layer is also clearly visible in the density profiles, which is discussed later on.

For the spin $\omega_{z}$ we also find a good agreement, see Figure 6 . In the middle of the flow field, both methods give the same mean spin. Close to the wall minor differences occur, 


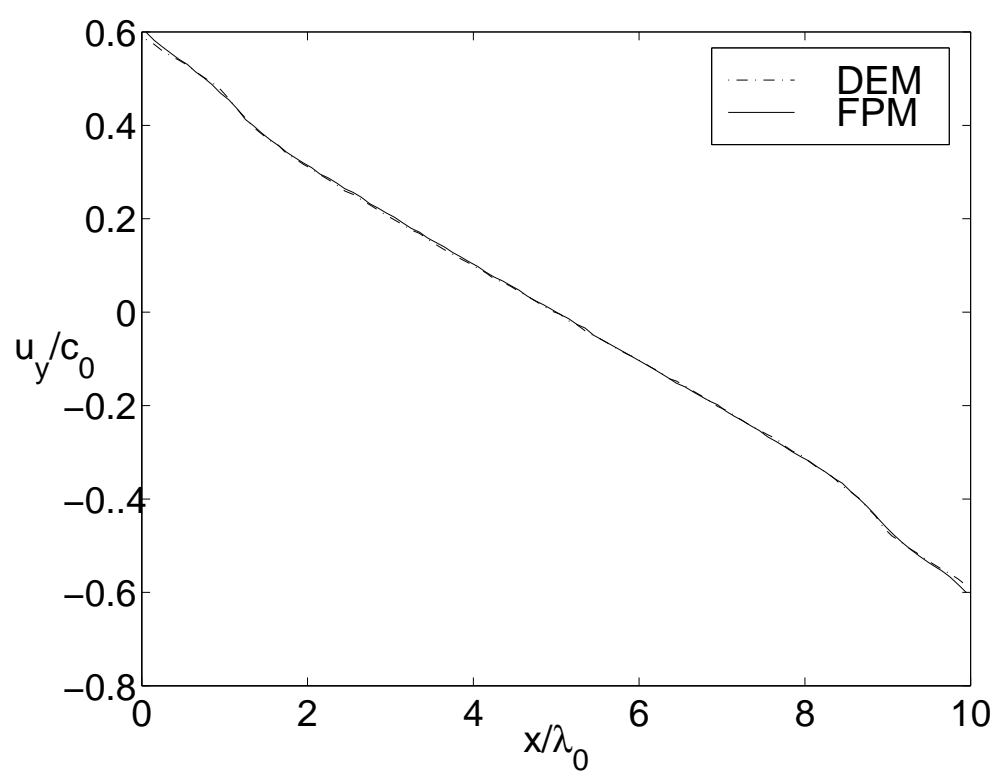

Figure 5: Mean velocity profile $u_{y}$ parallel to the walls for the FPM and the DEM, both for a $2 \mathrm{~d}$ velocity space.

where the DEM profile is slightly lower than the FPM one. It is not clear which one is more correct.

In Figure 7 the density profiles show a highly damped oscillation close to the wall which is characteristic of dense gases [10]. The reason for this is that some part of the surface of a particle close to the wall is shielded from collision. Hence, particles which are situated close to the wall are pushed even closer against it; they hardly get a chance to re-enter the flow. The large increase in density close to the wall with a less dense zone separating it from the central uniform density core is a distinctive feature of dense particle ensembles. The variation of the density occurs in a region of the order of a particle diameter and becomes smaller as $\nu_{0} \rightarrow 0$. In the Boltzmann limit the density becomes spatially uniform.

Again, the density profiles of both the DEM and the FPM agree very well. The only significant, but still small difference between them is the local maxima in the FPM solution which lies just inside $2 a$ from the walls. The reason for this is likely to be that the equilibrium pair correlation $h^{(2)}$ has been used close to the walls, thus neglecting changes that are known to occur in $h^{(2)}$ close to the walls, see [18].

The energy profiles are shown in Figure 8 and compare excellently. The total energy $e_{\text {tot }}$ is defined as:

$$
e_{\mathrm{tot}}=e_{v}+e_{w}+\frac{1}{2} m \boldsymbol{u}^{2}+\frac{1}{2} I \overline{\boldsymbol{\omega}}^{2},
$$

which is the sum of inner and macroscopic energies. All the energy profiles are scaled by $\frac{3}{4} m c_{0}^{2}$, which is the inner translational energy for a hard sphere gas with most probable 


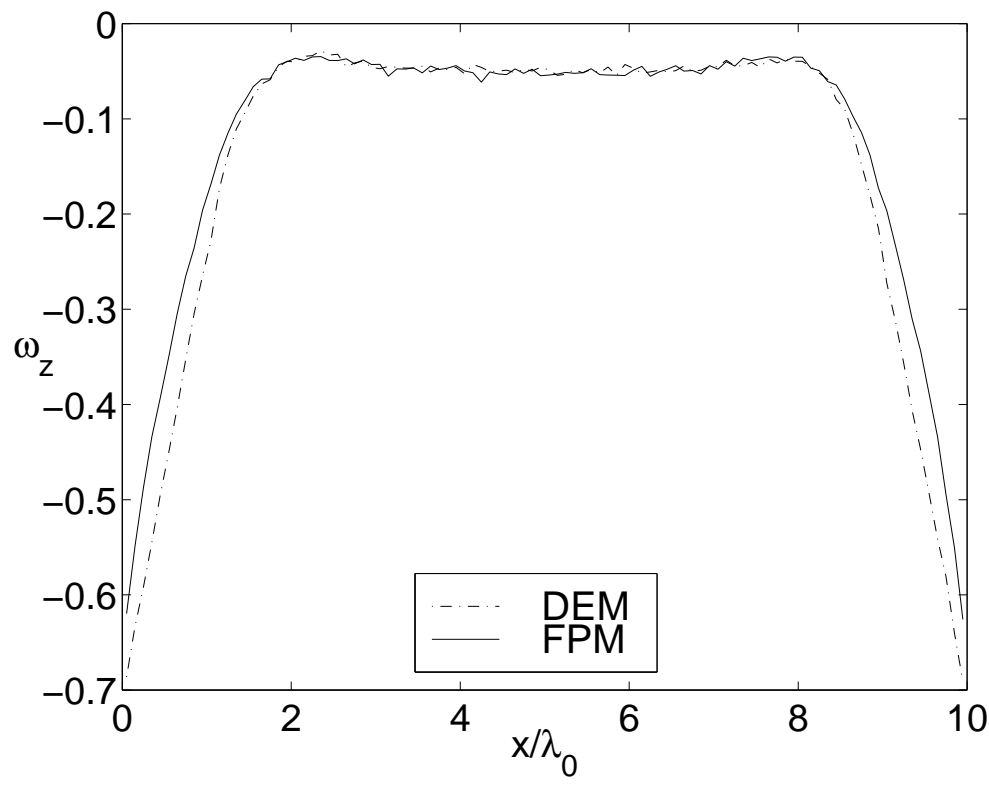

Figure 6: Mean particle rotation profile $\omega_{z}$ shows good agreement for the FPM and the DEM in $2 d$.

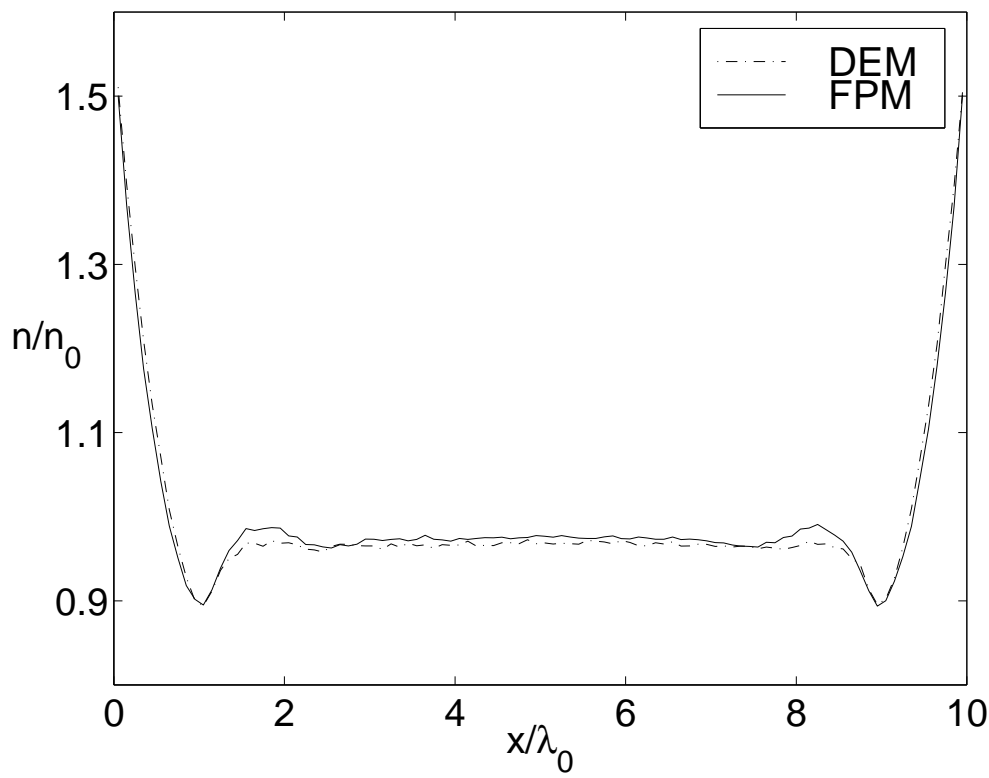

Figure 7: Density profiles for the FPM and the DEM, both for a $2 \mathrm{~d}$ velocity space.

speed $c_{0}$. The inner energy profiles are close to relaxation, in the sense that the inner energy is equi-partitioned over all three degrees of freedom, $e_{v} \approx 2 e_{\omega}$. This is consistent 
with there being two translational but only rotational degree of freedom. This means that the amplitude of the velocity fluctuations are the same in each spatial direction and also for the spin.

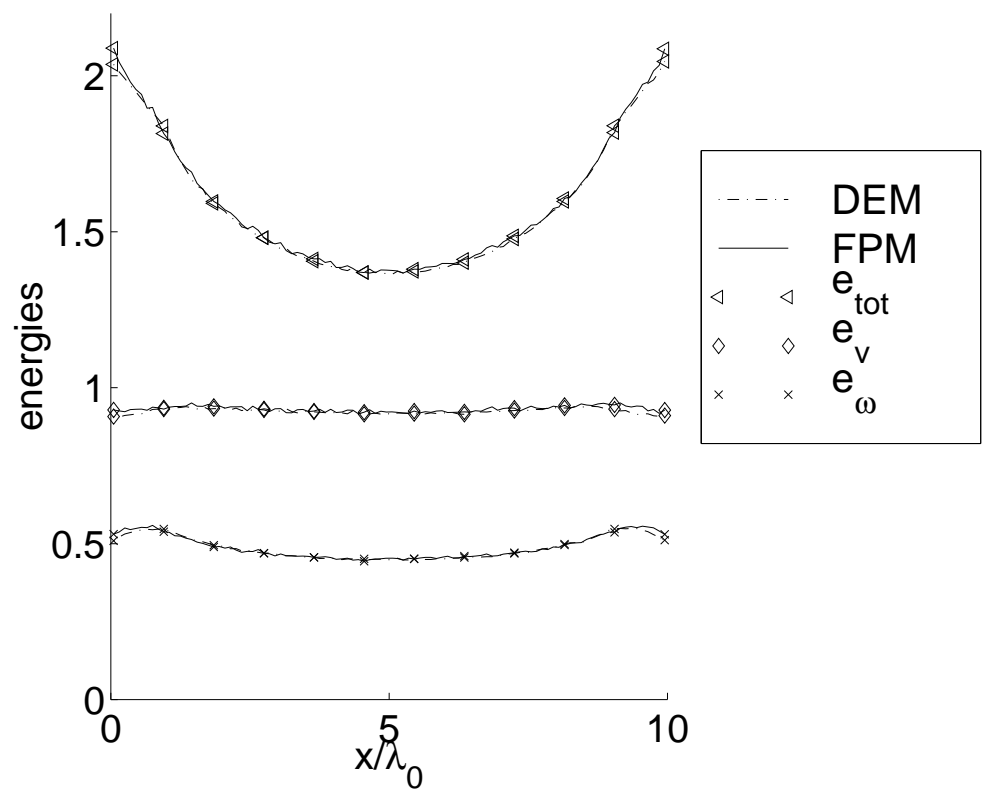

Figure 8: Energy profiles for the FPM and the DEM, both for a 2 d velocity space.

The comparison of DEM and FPM show that they, in the $2 \mathrm{~d}$ case, lead to the same flow pattern, density profile and macroscopic moments, with only very small differences. In the following we compare the FPM in a $3 \mathrm{~d}$ velocity space to the $2 \mathrm{~d}$ DEM and see that, although some differences are noticeable, both methods qualitatively deliver the same results.

\section{Comparison of 3D FPM to 2D DEM}

In the comparison of the FPM method operating on a velocity space with three translational and three rotational degrees of freedom, the proper choice of the number densities $n_{2 d}$ and $n_{3 d}$ (or solid fractions $\nu_{2 d}$ and $\nu_{3 d}$ ) in the $2 \mathrm{~d}$ and $3 \mathrm{~d}$ test cases is of high importance.

The geometric considerations of $2 \mathrm{~d}$ close packing establish an upper bound for the solid fraction of $\nu_{2 d} \leq \nu_{2 d, \max }=\frac{\sqrt{3}}{6} \pi \approx 0.907$. Similarly, the hexagonal close packing in $3 \mathrm{~d}$ yields $\nu_{3 d} \leq \nu_{3 d \text {,max }} \approx 0.74$. The kinetic granular flow equation (1) is not valid for solid fractions close to the maximal solid fraction. However, the different maximal solid fractions for two and three dimensions show that it is not sensible to simply set $\nu_{2 d}=\nu_{3 d}$ in the $2 \mathrm{~d}$ and 3d simulations and still expect similar results.

In the previous section we saw that when the mean free path $\lambda$ is of the order of the 
diameter $a$ of the spheres, then the density profile develops a peak close to each wall. This phenomenon is independent of the spatial dimension, hence we assume that similar (but not equal) density profiles are obtained, if

$$
\frac{\lambda_{2 d}}{a}=\frac{\lambda_{3 d}}{a} .
$$

Using Eqs. (30) and (31), we find

$$
\nu_{2 d} h_{2 d}^{(2)}\left(\nu_{2 d}\right)=\frac{3}{4} \pi \nu_{3 d} h_{3 d}^{(2)}\left(\nu_{3 d}\right) .
$$

In particular, for $\nu_{3 d}=0.1$ the diameter of a sphere is $a \approx 1.106 \lambda_{3 d}$. Using (39), the corresponding solid fraction for a $2 \mathrm{~d}$ translational velocity space is then $\nu_{2 d} \approx 0.213$. For each scheme the distance between the walls is set to $10 \lambda+a$. This guarantees that both schemes develop the characteristic density peaks close to the walls. However, we cannot expect that they will be identical. The reason for this becomes clear when we consider the energy distribution in the system. The inner translational energy $e_{v}$ is responsible for the collision frequency. If the inner energies were totally relaxated, then the total inner energy would be evenly distributed over all degrees of freedom. For this DEM implementation this means that the inner translational energy $e_{v}$ is twice the inner rotational energy $e_{\omega}$ (as the translational velocities are $2 \mathrm{~d}$ but the spin is only $1 \mathrm{~d}$ ). On the other hand, in the FPM method $e_{v}=e_{\omega}$, since there are three translational and three rotational degrees of freedom. The partitioning of the total inner energy to the various degrees of freedom is different for both schemes and hence the collision frequencies are different as well.

Deviations in the energy profiles produce deviations in the density profiles because the particles accumulate in the 'colder' central core of the shear flow.

The stationary profiles for the bulk velocity and the spin agree these two methods agree very well, despite the different velocity spaces in which they are operating. The curves are not shown here since the $3 \mathrm{~d}$ FPM profiles are essentially the same as the $2 \mathrm{~d}$ ones, given in Figures 5 and 6.

As was already mentioned above, different dimensions of the velocity spaces mean that we cannot expect identical energy profiles for both methods. Figure 9 shows the profiles of the total energy $e_{\text {tot }}$, as defined in (37), the inner translational energy $e_{v}$ and the inner rotational energy $e_{\omega}$. All the energy profiles are scaled to $\frac{3}{4} m c_{0}^{2}$, which is the inner translational energy for a hard sphere gas with most probable speed $c_{0}$.

The inner energies $e_{v}$ and $e_{\omega}$ of the FPM are very close to each other. As both energy forms have the same number of degrees of freedom, namely three, the profiles show that the flow is nearly relaxated. The inner energies found in $2 \mathrm{~d}$ are qualitatively very similar to those found in $3 \mathrm{~d}$.

The difference between the $e_{v}$ and $e_{\omega}$ profiles for the FPM is larger close to the boundary. This is due to the shearing of the walls adds energy to the flow in this region and the roughness of the particles then produces large changes of the spin leading to higher inner rotational energy in this region. 


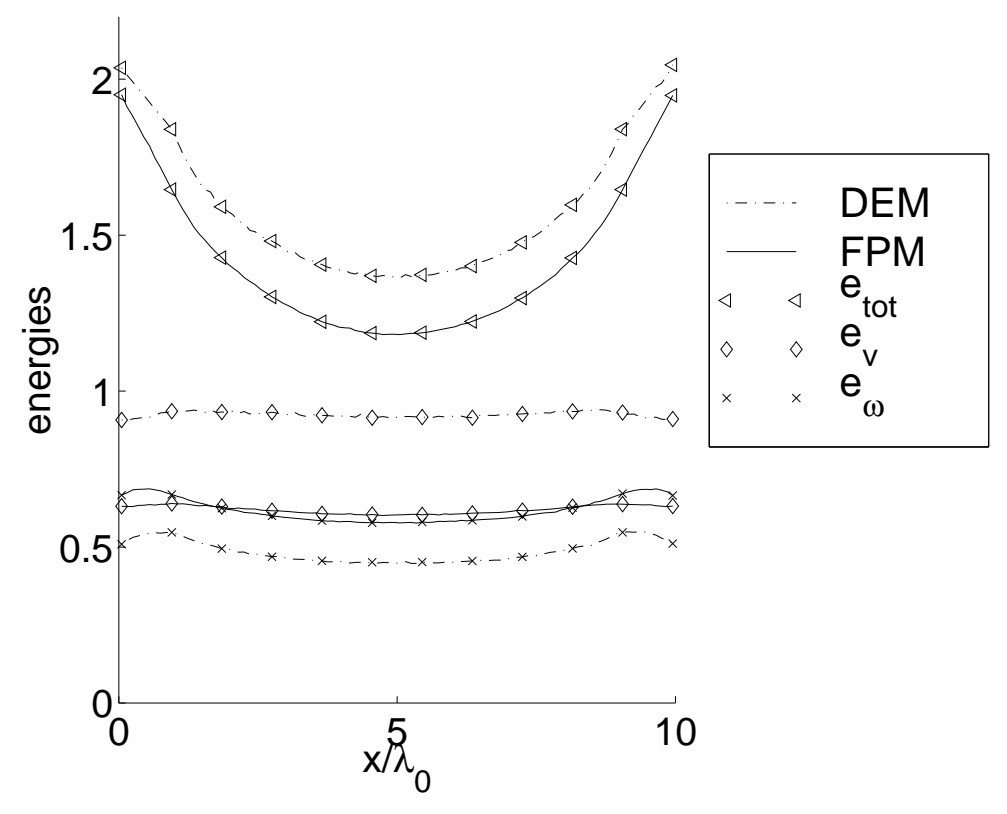

Figure 9: Energy profiles for the 3d FPM and the 2d DEM.

The inner energy profiles of the DEM are also close to relaxation with $e_{v} \approx 2 e_{\omega}$, after taking account of the differing number of degrees of freedom. As with the FPM, $e_{\omega}$ has a local maximum close to the wall.

The total energy profile derived from the FPM is around $15 \%$ lower than the respective DEM profile. Dimensional differences again produce this deviation in magnitude and were described in earlier on. Importantly, the shape of the energy profiles is the very similar.

The differences in the inner translational energies between methods naturally leads to deviations in the number density profiles, shown in Figure 10. The gradient of total inner energy in the boundary layers is slightly larger for the FPM than for the DEM. This leads to to a deeper U shape for the inner energy with relatively lower levels of inner energy in the center of the channel for the FPM. Lower inner energy means lower collision rates, leading to smaller particle collisional pressures and therefore higher densities. This is clearly visible in Figure 10 by the slightly higher central density and slightly lower boundary layer densities for the FPM. The difference in the inner energy profile has caused more particles to accumulate in 'colder' central regions of the flow.

The difference in the energy profiles are not the only reason for the differences in the density profiles. Here again, the difference in the dimensions has an important influence on the profiles. However, both profiles are qualitatively similar and show the peak in density at the boundary of the flow domain and a minimum at the distance of a diameter. The comparison of DEM and FPM has shown that they lead to the similar macroscopic moments, with only small differences. The dimension of the velocity space plays a modest 


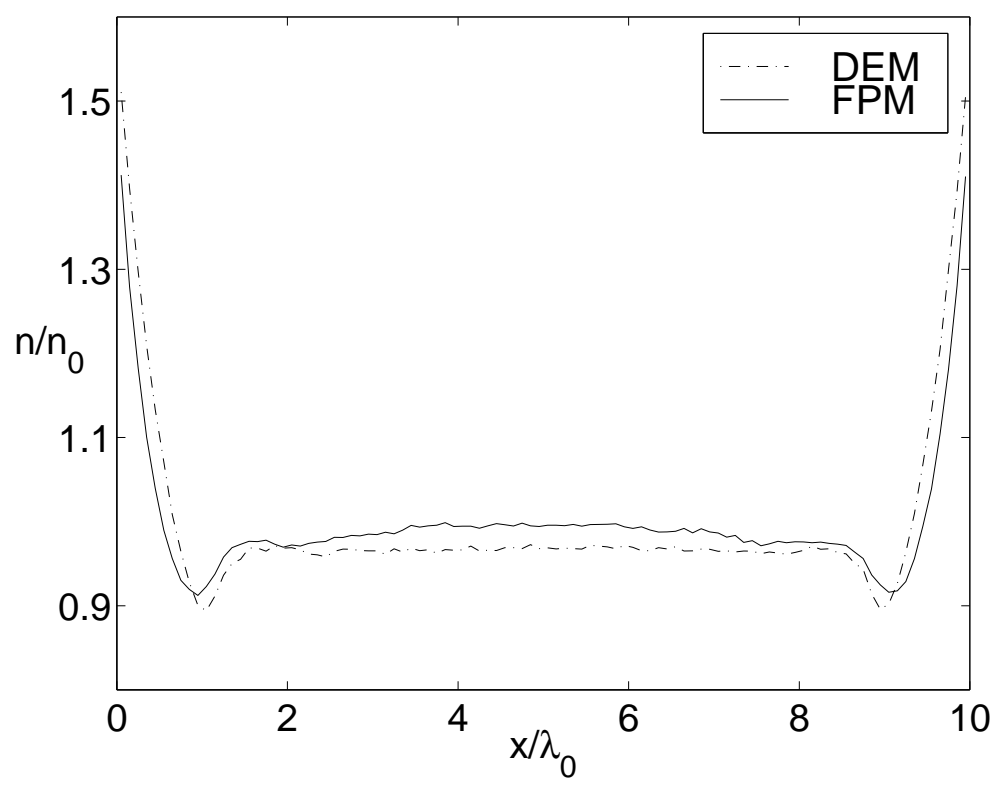

Figure 10: Density profiles for the 3d FPM and the 2d DEM.

but important role in the distribution of the inner energy between the various degrees of freedom and thus on the energy and density profiles. The fact that the present DEM implementation only uses a reduced velocity space, has to be kept in mind if industrial problems are modelled. The closeness of the velocity and density profiles, however, suggests that the use of the $2 \mathrm{~d}$ DEM to model $1 \mathrm{~d}$ and $2 \mathrm{~d}$ granular flows is unlikely to introduce significant errors.

In the next section, computational times of both methods are compared. It is shown that, for dilute and moderately dense ensembles, the FPM is much faster than the DEM.

\section{Comparison of Computation Times}

To determine the efficiency of the DEM and the FPM, we consider the CPU-time that is consumed for the simulation of a time interval of length $\Delta T$. This certainly depends on the number of particles in the system. Where the DEM for the Couette flow delivers reasonable results for as few as 10 particles, the FPM needs at least a handful of particles in every cell with length $l$. The cell size for the FPM depends on the mean free path $\lambda$ because $l<\lambda$. If the distance between the walls in the Couette flow is $10 \lambda$, and the profiles are to be evaluated at, say, 100 different positions or at every $1 / 10$ of the mean free path, then the FPM needs at least a few hundred particles, where the DEM still uses 10. However, the 'noise' of the profiles also depends on the number of particles that is used for the averaging at a position or within a cell. For an objective comparison of efficiency, we compare the CPU-times for the simulation of $N$ particles per scaled time interval $T$. 
The DEM usually does not use more than a few thousand particles for its simulation, here we choose a maximum number of 1,000 discs.

In the following, four different settings are considered, namely two for the DEM, using a parameter ratio of $k_{\eta} / m=10,416$ and 1,042 . The resulting energy profiles are such that the mean overlap is around $0.8 \%$ and $2.4 \%$, respectively. The two settings for the FPM are distinguished by the calculation of the post-collisional velocities. In a first run, the post-collisional velocities are determined by solving the force equations for the springdashpot model as is done in the DEM, i.e. by a simple explicit scheme solving the system of ordinary differential equations, Eqs. (24)-(26). In a second run, the hard sphere collision model for the FPM with

$$
e=0.95, \quad \text { we set } \quad \beta=0.915 \text {, }
$$

as computed from the spring-dashpot model with friction coefficient $\mu=10^{6}$.

All runs simulate a Couette flow at a solid fraction $\nu_{3 d}=0.1$ for the FPM and matching $\nu_{2 d}=0.213$ for the DEM. The profiles are evaluated at 100 different positions of the total distance $L=10 \lambda+a$ between the walls.

The CPU-times per 100 time units simulation time running on a DEC 3000/600 are given in Figure 11. The number of particles in this DEM is varied by changing the length of the control volume along the plates, while keeping the number density constant.

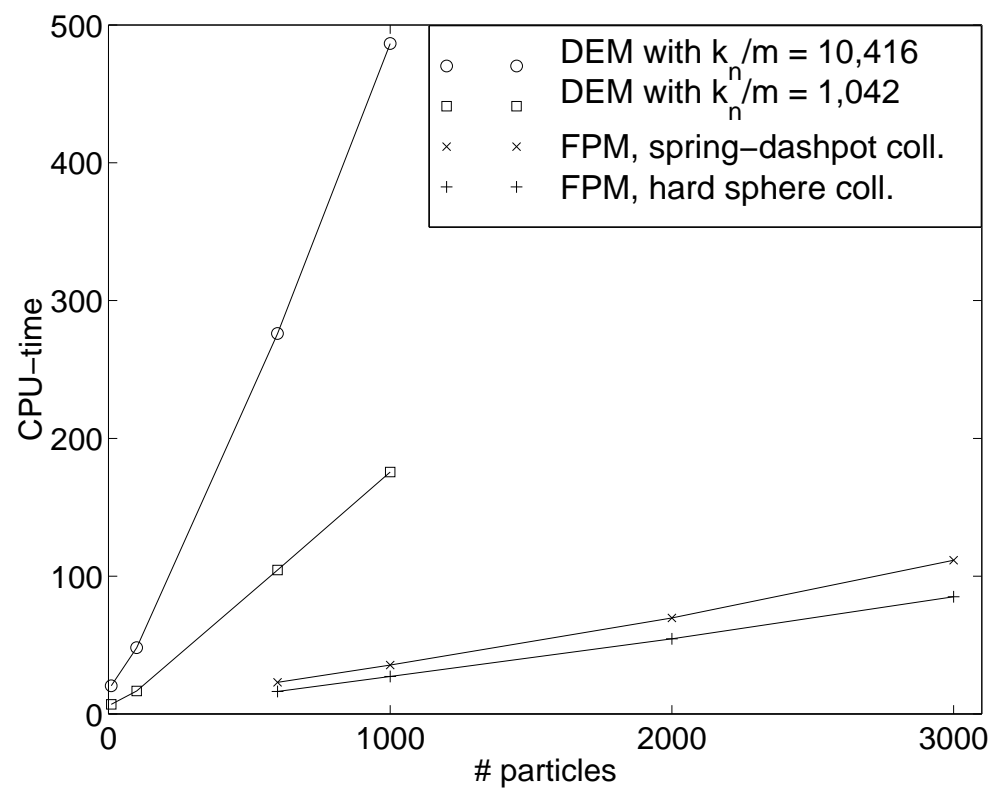

Figure 11: CPU-time for 100 time units simulation time, dependent on the number of particles.

The uppermost line, represented by ' $\circ$ ', shows the CPU-time for the DEM with a parameter ratio of $k_{\eta} / m=10,416 \mathrm{~s}^{-2}$, i.e. when the soft particle collisions are very close to 
hard sphere collisions, as shown in Figure 2. For more than 100 simulation particles, the dependence of the CPU-time on the number of particles is almost linear.

If the parameter ratio is reduced to $k_{\eta} / m=1,042 \mathrm{~s}^{-2}$, the particle collisions are softer and the time for a binary collision is a factor $\sqrt{10}$. Hence, the time step is larger and the CPU-time is reduced by this factor. We found that for a ratio of $k_{\eta} / m=1,042 \mathrm{~s}^{-2}$, the stationary profiles for the Couette flow do not vary more than $5 \%$ from the corresponding profiles for 'very hard soft collisions' with $k_{\eta} / m=10,416 \mathrm{~s}^{-2}$. Hence, if minor deviations are acceptable, one might also choose to run the DEM simulations with this larger time step. The corresponding CPU-time, depending on the number of simulation particles, is given by the line denoted by ' $\square$ ' in Figure 11. For even smaller parameter ratios $k_{\eta} / m$, the deviation from the hard sphere profiles is profound and are therefore not considered here.

On the other hand, the FPM is much faster. The FPM simulating the binary springdashpot encounter by solving the underlying soft particle collision model is denoted by ' $X$ ' in Figure 11. It shows that the CPU-time is a factor 5 faster than the CPU-time of the DEM with parameter ratio $k_{\eta} / m=1,042 \mathrm{~s}^{-2}$, or a factor 14 for a ratio of $k_{\eta} / m=$ $10,416 s^{-2}$.

The FPM based on the hard sphere collision model is slightly faster again, see the line denoted by the '+'-signs. The CPU-time is a factor 6 to 18 faster than the above mentioned DEM simulations. Of course, the hard sphere model with constant $\beta$ is not particularly well-suited, if the friction coefficient $\mu$ is close to one, since then $\beta$ actually depends on the pre-collisional velocities.

A word of caution is necessary with such comparisons. Not only do the DEM and FPM have different velocity spaces and therefore different calculation costs, but the implementations also use different languages. The DEM is written in FORTRAN 77, whereas the FPM implementation is a C-code. Different high level languages may, for the same problem, result in different computation times. This difference also depends on the platform. In addition, the DEM code has been optimized for dense flows and would be faster if optimized for modest density flows such as these. Despite these factors which will affect the specific speed factor differences, the underlying shapes of these curves will not be affected and we expect the FPM to still be quite a bit faster.

If the solid fraction $\nu_{3 d}$ increases, the mean free path becomes smaller. As a consequence, the discretization of the FPM has to be refined as well. In the FPM, for $\nu_{3 d}=0.1$ and $L=10 \lambda_{3 d}+a$, the mean free path is split into a fixed number of cells. (To improve readability, the subscript $3 \mathrm{~d}$ is omitted in the following and $\nu_{0}$ is set to $\nu_{0}=0.1$ ). If the gap between the walls is kept constant with $L=10 \lambda+a$ where $\nu=\nu_{0}$, then, for an increasing solid fraction, the number of cells in the whole domain increases also. For instance, $\nu=0.2$ gives $L \approx 27 \lambda+a$, increasing the number of cells by a factor of 2.7 .

The DEM does not have to resolve the mean free path, i.e. the number of particles necessary for the scheme is independent of the solid fraction. Hence, the CPU-time per unit time and for a fixed number of particles is nearly constant with respect to the solid frac- 
tion (it increases only slightly, since the number of neighbours increases with the solid fraction).

In Figure 12, the CPU-times of both schemes are compared for a varying 3d solid fraction (the corresponding $2 \mathrm{~d}$ solid fraction for the DEM is computed via (39)). The solid lines '-' are the interpolated curves. 1,000 particles have been used for the DEM for all solid fractions. For the FPM, 1,000 particles are used for $\nu=\nu_{0}$. For higher solid fractions, the number of cells increases. If the number of particles per cell is kept constant in the FPM to maintain consistent accuracy, the total number of particles in the system grows by a factor $\nu h^{(2)}(\nu) / \nu_{0} h^{(2)}\left(\nu_{0}\right)$.

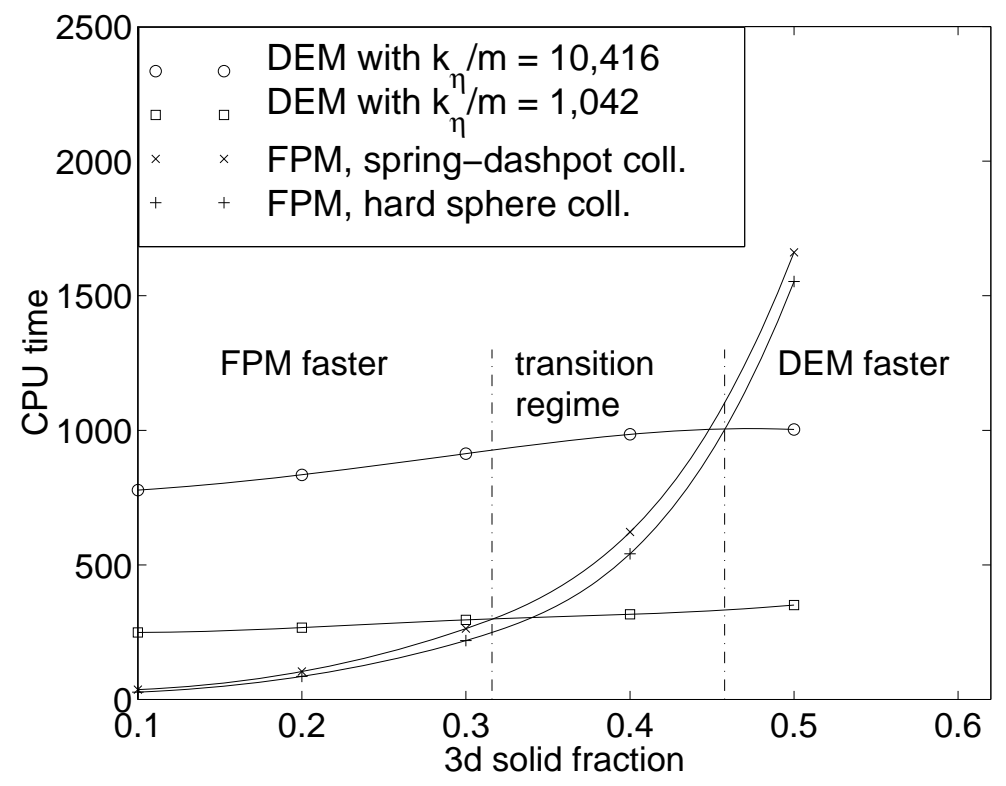

Figure 12: CPU-time for 100 seconds simulation time with varying solid fraction $\nu_{3 d}$.

In the moderately dense stage for $\nu \lesssim 0.32$, the FPM is always faster than the current DEM, even for the softer spheres with $k_{\eta} / m=1,042 \mathrm{~s}^{-2}$. For higher solid fractions in the transition regime $0.32 \lesssim \nu \lesssim 0.46$, the FPM, based on the hard sphere collision, is still faster than the DEM with $k_{\eta} / m=10,416 s^{-2}$, but slower than the DEM using the softer collisions. For $\nu \gtrsim 0.46$, this DEM is finally always faster than the FPM. This is a very important regime since all applications which include gravity have solid fractions which exceed this limit. These applications are ones for which DEM becomes a viable and preferred option for modelling technique. The inclusion of more degrees of freedom, such as particle size, shape and density distributions also strongly favour the DEM as the dimension of the space in which the FPM scheme operates rises strongly and sufficient particles must be available to statistically sample all these dimensions. 


\section{Conclusions}

A particle scheme for solving kinetic granular flow equations, based on finite pointset methods, and a discrete element method were compared using a homogeneous granular flow and a granular Couette flow. The macroscopic flow quantities (velocity, density and inner energy profiles) given by the two methods agree very closely for simulations in two dimensions. Three dimensional kinetic FPM solutions for the Couette flow were also compared with the two dimensional DEM, revealing qualitatively similar behaviour. The flow velocity and density profile are quantitatively close with moderate differences in the magnitudes of the inner energies. Most of the quantitative differences result from the difference in the dimensionality of the velocity spaces of the two solutions. The inner energy per degree of freedom was very similar for this case.

It is shown that the important criterion to make valid comparisons between two and three dimensional solutions is that the mean free path length of the two flows must be the same. Matching the solid fractions does not lead to valid comparisons, because the dynamics can then be quite different.

This suggests that the use of two dimensional simulations to solve for granular flows which are one or two dimensional in space gives results that are close to those obtained using a three dimensional scheme. This is important for the interpretation of the widespread two dimensional DEM simulations of geophysical and industrial granular flows.

For dilute and moderately dense particle ensembles, the FPM is faster than the discrete element method. For dense flows or flows with complex geometries or complex particle

distributions the FPM becomes increasingly expensive and the DEM becomes much more efficient.

\section{References}

1. B. J. Alder and T. E. Wainwright, Studies in molecular dynamics, J. Chem. Phys., 33, p. 1439 (1960).

2. G. C. Barker, Computer simulations of granular materials, in: Granular Matter: An interdisciplinary approach, Ed. Anita Mehta, (Springer-Verlag, NY, 1994), pp. 35-83.

3. G. A. Bird, Molecular Gas Dynamics and the Direct Simulation of Gas Flows, (Clarendon Press, Oxford, 1994).

4. N. F. Carnahan and K. E. Starling, Equation of state for nonattracting rigid spheres, J. Chem. Phys., 51, 635-636 (1969).

5. P. W. Cleary, Modelling industrial granular flows, Proc. 1st Australian Enginneering Mathematics conference, Melbourne, 11-13th July, Eds. A. K. Easton and J. M. Steiner, (Studentlitteratur, 1994), pp. 169-177. 
6. P. W. Cleary, Discrete element modelling industrial granular flows, Proc. 2nd Australian Enginneering Mathematics conference, Eds. W. Y., Yuen, Broadbridge, P., and Steiner, J. M., Institute of Engineers, Australia. Sydney, 15-17th July, pp. 301-308, (1996).

7. P. W. Cleary, Predicting charge motion, power draw, segregation, wear and particle breakage in ball mills using discrete element methods, 11, no. (11), Minerals Engineering, (1998).

8. P. W. Cleary, The Filling of Dragline Buckets, to appear: Mathematical Engineering in Industry, (1998).

9. D. Enskog, Kinetische Theorie der Wärmeleitung, Reibung und Selbstdiffusion in gewissen verdichteten Gasen und Flüssigkeiten, Kungl. Svenska Vetenskapsakademiens Handlingar, 63, pp. 1-44 (1922).

10. A. Frezzotti, A particle scheme for the numerical solution of the Enskog equation, Phys. Fluids, 9, pp. 1329-1335 (1997).

11. A. Goldshtein and M. Shapiro, Mechanics of collisional motion of granular materials. Part 1. General hydrodynamic equations, J. Fluid Mech., 282, pp.75-114 (1995).

12. A. Goldshtein, M. Shapiro, and C. Gutfinger, Mechanics of collisional motion of granular materials. Part 3. Self-similar shock wave propagation, J. Fluid Mech., 316, pp. 29-51 (1996).

13. H. Neunzert and J. Struckmeier, Particle methods for the Boltzmann equation, Acta Numerica, pp. 417-457 (1995).

14. L. Popken, Grid-free particle method for the inhomogeneous Enskog equation and its application to a Riemann problem, Eur. J. Mech. B/Fluids, 17, pp. 255-265 (1998).

15. L. Popken, Numerical Methods for a Kinetic Equation in Granular Flow, phd. thesis, (University of Kaiserslautern, 1998).

16. P. Résibois and M. D. Leener, Classical Kinetic Theory of Fluids, (John Wiley \& Sons, 1977).

17. I. C. Sanchez, Virial coefficients and close-packing of hard spheres and discs, J. Chem. Phys., 101, pp. 7003-7006 (1994).

18. I. K. Snook and D. Henderson, Monte Carlo study of a hard-sphere fluid near a hard wall, J. Chem. Phys., 68,5, pp. 2134-2139 (1978).

19. K. Steiner, Kinetische Gleichungen zur Beschreibung verdünnter ionisierter Gase und ihre numerische Behandlung mittels gewichteter Partikelmethoden, phd. thesis, (University of Kaiserslautern, 1995). 
20. J. Struckmeier and K. Steiner, A comparison of simulation methods for rarefied gas flows, Phys. Fluids, 7(11), pp. 2876-2885, (1995).

21. O. R. Walton, Numerical simulation of inelastic frictional particle-particle interaction, Chapter 25 of Particulate two-phase flow, pp. 884-911, ed. M. C. Roco, (ButterworthHeineman, Boston, 1994). 